\title{
Profesores universitarios memorables, a través de sus relatos autobiográficos ${ }^{*}$
}

\author{
Marta Osorio de Sarmiento*
}

Recibido: 20 de enero de 2012 Evaluado: 17 de febrero de 2012 Aceptado: 26 de marzo de 2012

\section{RESUMEN}

Este artículo tiene el propósito de identificar la concepción que los profesores memorables tienen sobre la didáctica, con la intención de indagar sobre la valoración que ellos otorgan de manera particular a las acciones didácticas, que orientan sus procesos de enseñanza y de modo específico al conocimiento que poseen de manera sistemática y reflexiva sobre la didáctica en sus prácticas pedagógicas; logrando de esta manera reconocer los hechos que los identifican como profesores memorables.

\section{Palabras clave}

Profesor memorable, acciones didácticas, práctica pedagógica.

* Artículo de investigación realizado para optar por el título en posdoctorado en Narrativa y Ciencia, (2010-2011) y que corresponde al grupo investigativo denominado Organización y gestión escolar, inscrito en Colciencias (Colombia).

** Doctora en Pedagogía. Docente Investigadora de la Facultad de Educación de la Universidad Santo Tomás, Bogotá. Actualmente dirige el Doctorado en Educación. Correo electrónico: osoriomalaver@gmail.com 


\title{
Memorable University Professors, Through their Autobiographical Stories
}

\author{
Marta Osorio de Sarmiento
}

\begin{abstract}
This article aims to identify the understanding that memorable professors have of didactics. In particular, it intends to find out how they value the didactic actions which guide their teaching processes, and more specifically, it studies the systematic and thoughtful knowledge these professors have about their teaching didactics. This way, it gets to identify the actions that have made them become memorable professors.

\section{KeYWORDS}

Memorable professor, didactic actions, teaching practice.
\end{abstract}




\section{INTRODUCCIÓN}

Este artículo se inscribe en el proyecto de investigación del Posdoctorado en Narrativa y Ciencia (2010-2011), Organización y gestión escolar, autobiografías de profesores memorables, del grupo de investigación del Doctorado en Educación de la Facultad de Educación de la Universidad Santo Tomás, Bogotá (Osorio, 2011). Asimismo, este proyecto da inicio a la línea de investigación de Organización y gestión escolar, con la intención de identificar a los profesores memorables. El contexto investigativo de este trabajo dispone a cuatro profesores de planta del Doctorado en Educación de la Facultad de Educación de la Universidad Santo Tomás, en los cuales se identificaron los elementos fundamentales que los catalogan como "profesores memorables" al analizar sus relatos autobiográficos.

El presente trabajo investigativo tiene como propósito identificar la concepción que los profesores memorables tienen sobre la didáctica; esto con la intención de indagar acerca de la valoración que ellos otorgan, particularmente, a las acciones didácticas que orientan sus procesos de enseñanza y, de modo específico, el conocimiento que poseen de manera sistemática y reflexiva sobre la didáctica en sus prácticas pedagógicas. Así se logra reconocer los hechos que los identifican como profesores memorables; es preciso señalar que se consideran estos elementos como incidentes en la pertinencia del ejercicio del profesor, es decir, en la calidad de sus prácticas en el campo de la educación superior y en sus logros y formación profesional.

Para ubicar a los profesores memorables se aplicaron entrevistas semiabiertas a los profesores del Doctorado en Educación, que incluyen: a) esbozo biográfico general, b) acceso a la enseñanza y ejercicio profesional, c) reflexión sobre la práctica pedagógica, d) reflexión sobre su conocimiento pedagógico, y e) reflexión sobre la mejora de la enseñanza.

Por medio de las siguientes preguntas se organizaron las ideas para plantear la discusión de quiénes son considerados profesores memorables. La intensión que se tiene es la de reconocer aquellos aspectos significativos que están en relación con la excepcionalidad de los profesores objeto de esta investigación.

- ¿Cómo influyen los profesores memorables y la familia en sus prácticas pedagógicas?

- ¿Qué acciones hacen que un profesor sea memorable?

- ¿De qué manera el conocimiento sobre la didáctica incide en la práctica de los profesores memorables?

- ¿Qué impacto académico se busca en los estudiantes por parte de un profesor memorable?

Mediante las entrevistas se buscó reconocer en los profesores sus experiencias formativas como estudiantes y luego como profesores en los diversos niveles de escolaridad en los que se han desempeñado. Asimismo, detectar los distintos elementos subyacentes y las representaciones adquiridas sobre su saber didáctico integrado al aula y la influencia de aquellos profesores memorables que a su vez ejercen efectos imperceptibles en sus prácticas de enseñanza. 
En este orden de ideas, las entrevistas se elaboraron con el propósito de indagar sobre hechos, eventos y vivencias de la vida personal, escolar y profesional de los profesores memorables, bajo la intención de encontrar relatos en los cuales se podían reconocer personas, profesores, mentores, instructores, hechos y experiencias significativas que han dejado huella y tienen influencia en formación académica y profesional en los cuatro profesores del doctorado en educación de la Facultad de Educación de la Universidad Santo Tomás; en este sentido se presentan los resultados de los cuatro profesores en mención, identificados como memorables: José Arles Gómez, de pedagogía (PM1), José Duván Marín Gallego, de epistemología (PM2), Mauricio Montoya, de filosofía (PM3), Claudia Vélez de la Calle, de derechos humanos (PM4).

El presente artículo se realiza desde el enfoque biográfico narrativo, como método de investigación para abordar las percepciones que los cuatro profesores memorables del Doctorado en Educación de la Universidad Santo Tomás, Bogotá, poseen de la didáctica, en tanto esta coadyuva a desarrollar sus prácticas pedagógicas. Se ha recurrido a las historias de vida como técnica de investigación, pues a través de ellas se logra recabar en la subjetividad de los individuos para alcanzar los hechos significativos de su vida profesional, o igualmente a través de las historias de vida se restablece el contenido emocional de las diferentes formas de experiencia humana velado por los métodos objetivos de un informe Booth (1996), en este sentido autores como Bolívar et ál. (1998) estima que:

Estos relatos que la gente cuenta sobre la vida personal o docente hablan de lo que hacen, sintieron, les sucedió o las consecuencias que ha tenido una acción; siempre contextualmente situados le sucedió o las consecuencias que ha tenido una acción, siempre contextualmente en relación con otros; no desde un yo solitario e imparcial. La narrativa expresa la dimensión emotiva de la experiencia, la complejidad, relaciones y singularidad de cada acción: frente a las deficiencias de un modo atomista y formalista de descomponer las acciones en un conjunto de variables discretas (p. 12).

\section{¿Quí́N ES UN PROFESOR MEMORABLE?}

Los notables cambios y transformaciones que está sufriendo el mundo actualmente han generado unas fuerzas de gran impacto para la inteligencia humana, que han afectado los diversos campos del saber, y de hecho los espacios académicos en los cuales estos acontecimientos adquieren protagonismo para quienes se relacionan con ellos, estudiantes y profesores, afectando de manera particular las prácticas pedagógicas de estos últimos.

Ante esta situación, es importante reconocer el papel protagónico que tienen algunos profesores en el campo del saber y la enseñanza, identificándolos a partir de sus prácticas pedagógicas (García-Valcárcel, 2001, p. 10), en tanto que estas las podemos reconocer como prácticas del conocimiento $\mathrm{y}$ es a ellas a las que se hace referencia cuando se reconocen que tienen un papel fundamental, para lo que ocurre en la sociedad actualmente. Así, para la intención de este artículo, es importante identificar las características de un profesor memorable que desempeña un papel fundamental en la formación universitaria. 
La proximidad que de una $u$ otra manera tenemos con nuestro mundo social, familiar o individual, el deseo de conocerlo, de comprenderlo, de explicarlo, de resignificarlo, se convierten en intenciones y acciones que dan origen a nuestras representaciones sobre este, sobre nuestros actos tangibles e intangibles, sobre las representaciones que tenemos de los demás y de sus actos, de las cuales emerge un conocimiento de la subjetividad dada en un contexto y su circunstancia histórica. En este sentido, la representación adquiere un valor singular en la interpretación, comprensión y conocimiento de los profesores en sus relatos autobiográficos, en términos de Bolívar et ál. (2001):

si los relatos autobiográficos no pueden ser vistos como un reflejo de la realidad, deben considerarse como representaciones hechas posibles por medio de los mecanismos discursivos del lenguaje, que los configuran como un relato (pp. 69-70).

Tratar el tema de la caracterización del profesor memorable, implica la percepción de este tipo de educador desde la representación que se puede tener de sus prácticas pedagógicas, de su personalidad, por las huellas que han dejado en las vidas de sus estudiantes, por su entusiasmo hacia el conocimiento, pero particularmente se identifica a estos profesores memorables por lo que se denomina actualmente como "buena práctica"; de acuerdo con Fenstermacher y Richardson comentado por Luis Porta et ál. (2009):

La enseñanza de calidad no solo produce aprendizaje a un nivel de razonable competencia - es decir enseñanza exitosa - sino que también se requiere que el contenido sea apropiado moralmente defendible, sostenido por concepciones de razonabilidad compartidas y según estándares disciplinares de adecuación y abarcabilidad (p. 2).

Según Porta, al hablar de profesor memorable, se hace referencia a su desempeño académico en términos de una buena enseñanza, esta se relaciona directamente con la acción de aprender, por parte del estudiante; pero también se puede considerar la noción que presenta Abdoulaye (2003), para quien las características de una buena práctica se orientan en dos ámbitos: en lo que el profesor debe realizar para que la actividad sea considerada una buena práctica y en los resultados que puede tener en los estudiantes. En la definición de Pere (2002), entendemos por buenas prácticas docentes las intervenciones educativas que facilitan el desarrollo de actividades de aprendizaje en las que se logran con eficiencia los objetivos formativos previstos y también otros aprendizajes de alto valor educativo, como por ejemplo una mayor incidencia en colectivos marginados, menor fracaso escolar en general, mayor profundidad en los aprendizajes. La bondad de las intervenciones docentes se analiza y valora mediante la evaluación contextual.

Al examinar la idea de algunos autores, sobre el concepto de buena práctica, se encuentra que ella se relaciona directamente con la didáctica, ya que la didáctica ${ }^{1}$ como

1 En la Didáctica Magna, publicada en 1657, recogiendo un conjunto de influencias circulantes en su tiempo, Comenio sostenía: "nosotros nos atrevemos a prometer una Didáctica Magna, esto es, un artificio universal para enseñar todo a todos. Enseñar realmente de un modo cierto, de tal manera que no pueda menos que obtenerse resultados. Enseñar rápidamente, sin molestias ni tedio alguno para el que enseña ni el que aprende, antes, al contrario, con el mayor atractivo y agrado para ambos. Y enseñar consolide, no superficialmente, ni con meras palabras, sino encausando al discípulo a las verdaderas letras, a las suaves costumbres, a la piedad profunda. Finalmente, nosotros demostramos todo esto a priori, es decir haciendo brotar como de un manantial de agua viva raudales constantes de la propia e inmutable naturaleza de las cosas, las cuales, reunidas en un solo 
herramienta pedagógica posibilita el desarrollo y eficacia de los procesos de enseñanza y aprendizaje llevados a cabo por los profesores. La relación íntima entre buena práctica y didáctica se puede observar en el planteamiento que hacen Camilloni et ál. (2008):

El discurso didáctico habla de y se dirige a indeterminados sujetos: los docentes (maestros, profesores, enseñantes). Destinatarios de la propuesta, son, sin duda, individuos reales. Y por sobre todo, individuos. Con particularidades que devienen de sus historias personales y, en especial. De su formación y experiencias profesionales. Su práctica se desarrolla en un lugar geográfico, un momento histórico, una determinada institución, un cierto contenido, un grupo específico de alumnos, con nombres, edades, intereses y necesidades que le son propias (p. 63).

De esta manera, propuesta la relación entre didáctica y la buena práctica, emerge de esta, la existencia del profesor (a propósito del profesor memorable). Afirma, al respecto, López (2006):

Un profesor es quien busca la autenticidad, es un profesor que está en autoconstrucción permanente y que puede, por ello, contagiar y propiciar la construcción de sus estudiantes, su propio progreso en autenticidad el desarrollo de su afectividad y la orientación de su trabajo común - el docente y alumnos- hacia algo que valga la pena, hacia la consecución de genuinos bienes particulares y de un más humano y justo bien de orden entre todos los hombres sustentados en valores terminales (p. 217).

Estas ideas suponen la relación entre una buena práctica sustentada en un ejercicio

caudal (cursiva agregada), forman el universal artificio para organizar las escuelas generales" (Comenio, 1982). didáctico que permite el reconocimiento no solo de la imagen de un profesor memorable, sino indudablemente de una relación dialógica entre profesores y profesores, estudiantes y profesores. El profesor memorable es aquel en el que su buena práctica está acompañada de su saber práctico; en palabras de Fenstermacher y Richardson (1993), la buena enseñanza depende del buen raciocinio práctico. Se puede decir que un profesor memorable es aquel que es generador de una buena enseñanza. Esta idea, donde se destaca el plano moral de la enseñanza, ha sido cuidosamente estudiada por Fenstermacher y Richardson (2005). Otra caracterización complementaria que se viene planteando sobre los profesores memorables, es la presentada por Bain (2007), que afirma que los profesores memorables:
conocen su materia extremadamente bien [...] son consumados eruditos, ar- tistas o científicos en activo [...] están al día de los desarrollos intelectuales, científicos o artísticos de sus campos, razonan de forma valiosa y original en sus asignaturas, estudian con cuidado y en abundancia lo que otras personas hacen en sus disciplinas, leen a me- nudo muchas cosas de otros campos y ponen mucho interés en los asuntos generales de sus disciplinas: la historia, controversias y discusiones epistemo- lógicas (pp. 26-27).

Bain (2007, p. 47) continúa su estudio indicando cómo una práctica docente de excelencia es aquella que crea entornos para el aprendizaje crítico-natural a través de los cuales se genera interés en el proceso de aprendizaje; por tanto, estas situaciones estimulan el comportamiento del estudiante frente al conocimiento. Así, se puede apreciar que los docentes memorables toman distancia de aspectos formales relacionados con la enseñanza y el aprendizaje 
tradicionales, como pueden ser las notas, la aprobación, el conocimiento por el conocimiento, y otro tipo de valoraciones que no tienen gran incidencia en el estudiante; por el contrario, el profesor memorable busca orientar los procesos de formación, de enseñanza y aprendizaje al despertar el interés de los estudiantes, estimulando sus capacidades y desarrollando y fortaleciendo la confianza de ellos en sus propias capacidades.

\section{RELACIÓN ENTRE DIDÁCTICA Y LA BUENA PRÁCTICA}

Las ideas, los pensamientos y conceptualizaciones que existen actualmente sobre las prácticas de la enseñanza se constituyen en un campo de estudio relacionado con la didáctica; las prácticas de enseñanza incitan a examinar la relación entre las acciones de enseñanza y las diversas concepciones didácticas que se aplican en ellas, más aún hoy, que dichas prácticas se encuentran presionadas y obligadas a evolucionar y transformarse generando en este hecho una complejidad en el campo de la didáctica. Con respecto a esta circunstancia, Díaz (2009) señala que:

En un momento en el que todo el orbe se impulsan reformas educativas que, bajo la premisa de mejorar la calidad de la educación persiguen modificar la práctica docente, esta disciplina constituye un factor fundamental para desentrañar el sentido educativo y pedagógico (p. 17).

Este autor continúa señalando cómo la didáctica se ha convertido en la educación superior en un campo de formación docente fundamental, motivo de reflexión acerca de la importancia que tiene en la misma formación de docentes universitarios. Para comprender la categoría que caracteriza a los profesores memorables, es importante hacer una revisión conceptual sobre la categoría de "didáctica"; a este respecto, llama la atención la propuesta que hace Benedito (1987) cuando presenta dos propuestas de interpretación del término, una de ellas desde el punto de vista etimológico: según el cual "la palabra 'didáctica' proviene del griego, más exactamente del verbo didasko $(\delta \imath \delta \alpha \sigma \kappa o)$, que significa enseñar, instruir, exponer claramente, demostrar; continúa señalando otros términos también desde su origen etimológico como son:

\begin{abstract}
diaktikós ( $\Delta$ เ $\alpha \kappa \tau \imath \kappa o \sigma)$, apto para la docencia, didaktiké $(\Delta t \delta \alpha \kappa \tau \imath \kappa \varepsilon)$, enseñando. didaskalia $(\Delta \imath \delta \alpha \sigma \kappa \alpha \lambda \iota \alpha)$, enseñanza, con una doble acepción en el campo de la enseñanza y en el teatro. Prosigue de acuerdo con la etimología del término didaktiko ( $\left.\delta \_\delta \alpha \kappa \imath \iota \kappa \sigma\right)$, indicando que la didáctica se puede definir como "la ciencia o el arte de la enseñanza" (Benedito, 1987, p. 7).
\end{abstract}

En la otra propuesta, señala que:

la multiplicidad de puntos de vista sobre lo que es y debe ser la didáctica, si bien pondrá de relieve divergencias más o menos profundas, conducirá al estatuto epistemológico de la didáctica y a su ubicación dentro de las ciencias de la educación (Benedito, 1987, p. 7).

Con esta afirmación se muestra su intención de plantear la fundamentación epistemológica de la didáctica basada en las corrientes epistemológicas contemporáneas, para tal propósito se centra en Pérez Gómez (1978 y 1982) y Bunge (1969, 1972, 1980,1985a y 1985b); Benedito nos hace pensar que la didáctica es un campo amplio que ha recibido y continúa recibiendo aportes de diversas disciplinas científicas, por ello adquiere diferentes formas y significados, además de 
estar en continua evolución; un ejemplo de ello es lo planteado por Pérez Gómez (1982, p. 118), para quien la didáctica en tanto ciencia aplicada, no solamente entra en discusión como tal, sino que debe conquistar un espacio propio dentro de la clasificación del campo científico; sin embargo, la didáctica se fundamenta en otras ciencias, pero a pesar de ello está en capacidad de producir conocimiento teórico sobre sí misma. De acuerdo con estas apreciaciones se puede afirmar que la didáctica establece su objeto y finalidad según su naturaleza científica, dando lugar a diversas formas de intervenir en las prácticas pedagógicas. En la búsqueda de algunas conceptualizaciones relevantes sobre el significado del término didáctica, se encuentran algunas definiciones, entre las que tenemos la de Mattos (1963): "La didáctica es la disciplina pedagógica de carácter práctico y normativo que tiene como objeto específico la técnica de la enseñanza, esto es, la técnica de incentivar y orientar eficazmente a los alumnos en su aprendizaje" (p. 34).

Por otra parte, Klingberg (1972) afirma que: "la didáctica se refiere a las relaciones regulares entre el hecho de enseñar y el aprendizaje, y está por tanto más unida al proceso de instrucción" (p. 47). Asimismo, Labarrere (1988) considera que:

La didáctica es la disciplina pedagógica que elabora los principios más generales de la enseñanza, aplicables a todas las asignaturas, en su relación con los procesos educativos y cuyo objeto de estudio lo constituye el proceso de enseñanza-aprendizaje (p. 32).

Mientras que Nérici (1985) afirma que:

La didáctica es el estudio del conjunto de recursos técnicos que tienen por finalidad dirigir el aprendizaje del alum- no, con el objeto de llevarle a alcanzar un estado de madurez que le permita encarar la realidad, de manera consciente, eficiente y responsable, para actuar en ella como ciudadano participante y responsable (p. 65).

Zabalza (2004, p. 12) declara que la didáctica empieza en la práctica para construir la teoría que posteriormente interviene de nuevo en la misma práctica. En los conceptos rastreados sobre la didáctica, se observa cómo esta se encuentra constituida según los autores señalados, por aspectos teóricos, prácticos con un carácter normativo y en algunos casos técnicos, designados para intervenir en la actuación de los profesores, en sus prácticas pedagógicas. Igualmente se le confiere el papel de direccionamiento de estas, con el propósito expreso de incidir en la formación de los individuos; de esta manera, la relación entre las prácticas pedagógicas y la didáctica es manifiestamente estrecha.

Resulta necesario entonces, apreciar el papel que desempeña la didáctica en las buenas prácticas de los profesores, pues ella ha adquirido "configuraciones" 2 que le posibilitan su evolución y apoyan las diversas actuaciones de los profesores frente al conocimiento, a los procesos de enseñanza-aprendizaje $y$, por ende, frente a la formación de las nuevas generaciones. De acuerdo con Porta (2010), la didáctica en construcción ha adquirido nuevas formas y variaciones que no solo inciden en el actuar de los profesores sino que también se hace evidente que dichas variaciones favorecen sus buenas prácticas.

2 Este término es tomado de Porta, de su libro Docencia universitaria (2010, p. 31). Quien señala que entendemos por configuración didáctica: "la manera particular que despliega el docente para favorecer los procesos de construcción de conocimiento" (Litwin, 1998, p. 157). 


\section{El ENFOQUE BIOGRÁFICO NARRATIVO}

La investigación biográfico-narrativa, considerada como un enfoque metodológico cualitativo, mediante el cual se posibilita acometer el estudio del desarrollo profesional docente, implica, entre otros aspectos, el estudio de documentos, informes y relatos personales de los profesores; de esta manera, se constituye en "una perspectiva o enfoque propio, que permite construir legítimamente conocimiento en la investigación educativa" (Bolívar, Domingo y Fernández, 2001, p. 32).

El método narrativo posibilita a través de las historias de vida, el acceso a las experiencias, ideas, a las personas relacionadas con la vida de otras personas, a las trayectorias escolares, personales y profesionales de las personas, en la intención de reconstruir, develar e interpretar su vida profesional, para dar significado en un pasado con referencia a un presente. A este respecto, Booth (1996), refiriéndose a la narrativa, señala que esta se asemeja a un retrato a través del cual se revelan las experiencias subjetivas de los sujetos en el sentido fiel que ellos confieren a sus propias vidas:

La multiplicidad de las formas que integran al espacio biográfico ofrece un rasgo en común: cuenta, de distintas maneras, una historia o experiencia de vida. Más allá del género en cuestión, se trata de una de las grandes divisiones del discurso - la narrativa - que está sujeta, por lo tanto, a ciertos procedimientos compositivos. Entre ellos, y prioritariamente, se encuentran los que remiten al eje de la temporalidad (Arfuch, 2008). Es decir, el anclaje imaginario en un tiempo ido, fantaseado, actual o prefigurado (Álvarez, 2009, p. 8).
Por otro lado, las historias de vida como recurso a través del cual se puede averiguar sobre la trayectoria de vida de los profesores, son reconocidas como:
Una metodología [...] para el estudio de las vivencias y el trabajo del pro- fesorado en un contexto social más pleno, con la intención de "desarrollar nuevas interpretaciones, a menudo de tipo colaborativo, acerca de la cons- trucción social del proceso de enseñan- za" (Goodson, 2004, p. 33).

Luego entonces, las historias de vida como un proceso de investigación narrativa inscrito en el giro hermenéutico de las ciencias sociales y humanas, pueden ser comprendidas como "textos cuyo valor y significado primariamente viene dado por la autointerpretación que los sujetos relatan en primera persona" (Bolívar, 2001, p. 15). Ellas aportan a la producción de conocimiento en el vasto campo de la educación, un saber profesional que surge del propio docente, pues el relatar, de alguna manera implica poseer un conocimiento subyacente en la vida académica y profesional del profesor, de su ambiente sociocultural, de unas experiencias que haciendo parte del pasado se presentan ante el presente como referente, y de acuerdo con la evidente relación entre relato y temporalidad, dirá Ricoeur (1999): “la narratividad y la temporalidad se encuentran estrechamente vinculadas, tan férreamente como puedan estarlo" (p. 183); más adelante, este mismo autor dirá sobre esta relación expresa, que el relato, en tanto configuración narrativa, acaba en una prefiguración de la experiencia temporal.

Entendemos como narrativa, por otra parte, la cualidad estructurada de la experiencia entendida y vista como un relato; asimismo (como enfoque de investigación), las pautas 
y formas de construir sentido a partir de acciones temporales personales, por medio de la descripción y análisis de los datos biográficos. Es una reconstrucción de la experiencia, por la que, mediante un proceso reflexivo, se da significado a lo sucedido o vivido (Ricoeur, 1995).

Para el desarrollo de la investigación que tiene por objeto de estudio, los profesores memorables, se ha encontrado en la investigación narrativa la posibilidad de aproximación a esta clase de profesional, pues este tipo de investigación, permite adentrarnos de manera profunda en sus prácticas, en sus actitudes, en su saber pedagógico, en la conceptualización que poseen sobre este saber; de manera que, con base en esta apreciación, es necesario señalar que se concibe de un lado a la narrativa, como la estructuración de la experiencia entendida y apreciada como un relato; de otro lado, como enfoque investigativo, las pautas y formas de construcción del sentido, con base en las experiencias temporales, mediante la descripción y el análisis de datos biográficos. De acuerdo con Ricoeur (1995), la narrativa es la reconstrucción de la experiencia, por la que, por medio de un proceso reflexivo, se da significado a lo sucedido o lo vivido. Además, el enfoque biográfico, como lo afirma Álvarez (2009):

Por su parte, destaca dos conceptos fundamentales como plataforma para su elaboración: el relato de vida -o narración de una vida tal como se ha vivido o es contada - y la historia de vida, que agrega a los relatos de vida las elaboraciones de los investigadores, así como otros registros y fuentes que validan la historia (p. 8).

Conviene declarar que la categoría "profesor memorable" tiene para esta investigación, como antecedente, las investigaciones realizadas por Álvarez, Porta y Sarasa
(2008-2010). Dichos investigadores, quienes en sus diversos estudios refieren a Goodson (2003), el cual a su vez, en sus estudios realizados sobre las historias de vida, registra algunos elementos conceptuales con los cuales se puede identificar a un profesor al que califica como "profesor favorito", señalado también como modelo, o profesor a imitar, siendo este tipo de profesor, en términos de los investigadores citados, el "profesor memorable", objeto de esta investigación.

La forma de vida del profesor, privada y en el ámbito universitario, ejerce gran influencia relacionada con su concepción sobre la enseñanza y de hecho con el conocimiento que tiene de esta en tanto práctica (Goodson, 2003, p. 249). Estos hechos señalados aparecen en las entrevistas en profundidad aplicadas a los profesores memorables, dando crédito a su práctica. La trayectoria académica y el desempeño profesional del profesor Goodson (2003, p. 750), se lleva a cabo en ciclos de vida que marcan una gran influencia en el desempeño profesional. Los hechos pedagógicos y los hechos de la vida personal marcan los procesos de sus prácticas y estilos de enseñanza.

Ahora bien, para escudriñar tanto en la vida privada como en la vida profesional de los profesores dispuestos para realizar esta investigación, se ha aplicado la entrevista en profundidad, considerada esta como una técnica de campo más genuina en las historias de vida (Buendía et ál., 1998, p. 282; Malagón, 1999, p. 187). Además, como herramienta por medio de la cual la exploración para la recolección de datos obtenidos de las entrevistas, permite, en términos de Ettling (1998), una explicación detallada, integradora y personal, entre la recolección de los datos y el análisis de los mismos, además 
de alcanzar diversos grados de escucha; se busca, mediante este tipo de entrevista, desarrollar una metodología con el propósito de lograr la intuición, racionalidad, la expresión creativa y la sistematicidad, características propias de la narrativa.

Mediante la entrevista en profundidad, se busca la construcción de significados en un "ordenamiento o reorganización de los materiales seleccionados del mundo actual" (Dillard, 1982). Se han realizado las entrevistas, intentando disponer de una manera de recrear un mundo en particular, recolectando los datos que permiten clasificación y organización de los hechos y experiencias, para establecer procesos y regularidades invisibles en el curso de la vida de los entrevistados. En las entrevistas en profundidad a los docentes memorables de nuestro estudio indagamos acerca de lo siguiente:

- ¿Cómo influyen los profesores memorables y la familia en sus prácticas pedagógicas?

- ¿Qué acciones hace que un profesor sea memorable?

- ¿De qué manera el conocimiento sobre la didáctica incide en la práctica de los profesores memorables?

- ¿Qué impacto académico se busca en los estudiantes por parte de un profesor memorable?

\section{LOS PROFESORES MEMORABLES} A TRAVÉS DE SUS HISTORIAS DE VIDA

“Contar historias es algo más serio y complejo de lo que hemos percatado alguna vez" (Bruner, 2003, p. 16). De aquí la rele- vancia que ha adquirido la investigación narrativa sobre las historias de vida de los docentes como "espacio" desde el que se construyen caminos y se detectan posturas biográficas, de su actuar pedagógico. Al respecto, afirma Hernández (2004):
La historia de vida como devenir y como presente de esperanza o incer- tidumbre, como pasado que nos en- seña lo que somos, como proyecto de futuro (de equidad, mayor justicia, de emancipación, en suma) sigue estan- do ahí para ser construida. Se mueve entre el relato y la experiencia, entre la investigación y la narración. Como líneas rectas, o desde los fragmentos y los residuos. Explorando lo conocido, o buscando elementos, evidencias que "hablen de otra manera", que cuenten la historia de otra manera" (p. 1).

Las historias de vida pueden ayudar a identificar la figura del profesor memorable a través de entrevistas en profundidad, realizadas a los profesores memorables del Doctorado en Educación, partiendo de sus relatos de vida; analizaremos los distintos aspectos que los llevaron al crecimiento personal e intelectual. Es así como los hechos, las vivencias, las experiencias, son entendidas como: "textos cuyo valor y significado primariamente viene dado por la autointerpretación que los sujetos relatan en primera persona" (Bolívar, 2001, p. 15). Por otra parte, es importante destacar los rasgos que han dejado huella y que se vivencian en sus prácticas y en su propia identidad. Los primeros mentores están muy relacionados con figuras cercanas a la familia, los cuales incidieron de una $\mathrm{u}$ otra forma llevando a estos a un desarrollo profesional. Visto desde la óptica de las aulas, vemos cómo un grupo de mentores conformado por los profesores memorables han influido en sus procesos de investigación y de selección de 
nuevos programas académicos. Un nuevo grupo destacado en sus carreras académicas, que marcaron un horizonte profesional y que siempre se entremezclaron en las vidas personales de los profesores, hicieron que su profesión sea algo muy importante en sus vidas. Plantean Connelly y Clandi$\operatorname{nin}(1994)$ :

\section{Pensar la vida como una historia es una forma poderosa de imaginar quiénes somos, dónde hemos estado y hacia dónde vamos. Desde esta perspectiva, las personas viven y cuentan relatos sobre esas vidas. La gente actúa como personaje en sus relatos de vida y en los relatos de otros. Vivimos relatos. Cuando hablamos con otros acerca de nosotros mismos, contamos relatos de vida (pp. 149-150).}

Nos preguntamos si es posible identificar prácticas concretas que conformen el trabajo usual de profesores memorables o si es una cuestión de principios generales, opinión, tendencias, e interpretaciones que aplican en el enseñar y el aprender donde se ve la mediación de los profesores memorable en sus clases. Pero es importante ver cómo nuestra experiencia de investigación nos ha llevado a analizar que no solo existe una sola forma sino muchas de desarrollar el "arte de enseñar" (Litwin, 2008, p. 13). De la misma manera, afirma Bain (2007):

La magia no reside, no obstante, en ninguna de estas prácticas. No puedo hacer más hincapié en la simple pero magnífica noción de que la clave para comprender la mejor docencia no puede encontrarse en reglas o prácticas concretas, sino en las actitudes de los profesores, en su fe en la capacidad de logro de sus estudiantes, en su predisposición a tomar en serio a sus estudiantes y dejarlos que asuman el control sobre su propia educación, y en su compromiso en conseguir que todos los criterios y prácticas surjan de objetivos de aprendizaje básicos y del respeto y el acuerdo mutuo entre estudiantes y profesores (pp. 92-93).

\section{¿Cómo INFLUYen los PROFESORES MEMORABLES Y LA FAMILIA EN SUS PRÁCTICAS
PEDAGÓGICAS?}

Al inicio de la investigación se realizaron entrevistas, contándoles a los profesores entrevistados el tipo de investigación y el papel que ellos jugarían en la misma; ellos se mostraron interesados en "contar su historia de vida", y evocar la influencia de sus mentores, profesores memorables y la familia, esta autodescripción deja ver cómo marcaron un gran influjo en sus prácticas universitarias actuales. PM1 relato su propia biografía escolar primaria y familiar con especial entusiasmo, como el lugar donde vivió "experiencias significativas" para su vida profesional. Entre los aspectos más significativos de la primaria, el bachillerato y familiar.

PM1 recuerda a los profesores de la primaria:
Profesora Fabiola Fernández, por su sana emulación y formación integral. Bachillerato: profesor, Henry Ibáñez, formación en español y literatura. Además, mis padres, en especial mi madre que me inculcó el "amor por la lectura". Las materias de español, li- teratura y sociales fueron igualmente fundamentales.

PM2 destaca la figura de la madre como gran influencia en su vida profesional y el amor por la escritura.

PM2 recuerda la influencia de profesores y compañeros en primaria y secundaria: 
La influencia fue principalmente de mis profesores, creo que por eso la "vocación docente quedó muy arraigada en mí", porque admiraba mis profesores, en primer lugar, mi profesor de primero de primaria de nombre José María Benjumea, del cual recuerdo todavía con nitidez su imagen, su personalidad y su entrega esmerada a sus alumnos. De él aprendí mis primeras letras y el "gusto por el estudio y la lectura". Luego en el bachillerato, en el Seminario de la Consolata, estuve rodeado de los "mejores profesores del mundo", toda vez que la comunidad se esmeraba de nombrar como docentes los sacerdotes más capacitados de la comunidad venidos de diferentes países del mundo: Italia, España, Canadá, Argentina y, por supuesto, Colombia. Además, muchos de estos sacerdotes ya habían tenido una gran experiencia como misioneros en distintas partes del mundo, especialmente en África y en América Latina, de cuyas "experiencia y relatos de vida en esas regiones nos llenaban a todos los estudiantes de entusiasmo por la vocación".

La calidad de sus profesores mentores y la forma como sus profesores extranjeros relataban sus experiencias profesionales de diferentes lugares del mundo le motivaron por la lectura. En el caso del tercer entrevistado son varios los factores que influyeron.

PM3. Creo, por ejemplo, que mi padre era un intelectual, y sus gustos eran amplios: literatura, biología, ciencias, historia, astronomía, el arte; le gustaba mucho el cine, diversos tipos de música: jazz, clásica, country, "de plancha", salsa, merengue, los boleros, incluso el rock. Luego, esa capacidad de amplitud de sentidos y aproximaciones frente al mundo indudablemente se debe en gran parte a una influencia ejercida por él. Pero la docencia está incluida en la genética de la familia, mi papá y dos de sus hermanas fueron docentes toda su vida laboral, una prima lo es actualmente, y de otros familiares, se podría contar otros seis casos de personas vinculadas a la docencia en diversos ámbitos. Bueno, en la primaria recuerdo especialmente la influencia del profesor Horacio y Marco Tulio; en la secundaria, del profesor Gonzalo, por su fuerte "carácter político", su postura crítica frente a la sociedad y su férrea formación académica, era un profesor que siendo magíster a comienzos de los años noventa daba clases tanto en la universidad como en el colegio.

Reconoce la influencia desde temprana edad de su padre y en general de su familia; de igual forma la de sus profesores, por la influencia política, lo cual le influyó para elegir su doctorado años más tarde.

PM4. Para "los que más influyó fue mi familia donde leer y pensar eran muy importante. También la literatura, la política y la música". Se resalta la influencia de la familia por la lectura y el desarrollo de un pensamiento crítico.

\section{¿Qué aCciones, ACTITUdes, APTITUDES HACE QUE UN PROFESOR SEA MEMORABLE?}

\begin{abstract}
Algunos profesores memorables mencionan la influencia de sus maestros para la elección de sus carreras académicas. La respuesta siguiente demuestra la disciplina del profesor memorable.
\end{abstract}

PM1. La influencia más importante es la dada por un "llamado interno, una vocación muy fuerte", un deseo de ayudar a formar a los demás desde los valores y desde un nuevo cambio de conciencia. Externamente han influido algunos docentes, entre ellos los más importantes: la profesora Fabiola Fernández $\left(5^{\circ}\right.$ de primaria), el profesor Henry Ibañez ( $6^{\circ}$ y $7^{\circ}$ de bachillerato) y fray Nelson Londoño (Seminario Franciscano). 
Estudié en la Universidad San Buenaventura (1989), la carrera: Licenciatura en Filosofía; en la Universidad Javeriana, (1997) la carrera: Licenciatura en Teología; en la Universidad Manuela Beltrán (1996), Especialización en Educación y Orientación Sexual; en la Universidad Santo Tomás (1997), Especialización en Educación-Filosofía Colombiana; en la Universidad Santo Tomás (2001), Máster en Filosofía Latinoamericana; en la Pontificia Universidad Urbaniana, Roma, 2008: Doctorado en Teología; en la Universidad Santo Tomás y Católica de Córdoba (2011), el Posdoctorado en Narrativa y Ciencia.

Se identifica cómo este profesor memorable, demuestra una vocación muy fuerte, por dejar una huella en valores en sus discípulos.

En el caso de PM2, sin duda, se nota que hubo muchos profesores que influyeron en su vida, especialmente por sus conocimientos, por su profesionalidad y por su entrega a los estudiantes. Quizás se siente más fuerte la influencia en los primeros años de formación, él nos cuenta:

En filosofía influyeron mucho los jesuitas en la Javeriana; eran profesores muy preparados en las materias que enseñaban. Recuerdo con especial atención el profesor de Historia de la Filosofía, el padre Jaime Vélez Correa y el profesor de Ética, el padre Alfonso Llano, no solamente por lo que sabían sobre las materias que enseñaban, sino también "por su pedagogía y la didáctica". En la Universidad San Buenaventura, recuerdo al padre José Ignacio Vives, quien dictaba un seminario sobre Nietzsche, pero sobre todo, "por la forma agradable" de hacer comprensible la materia.
Muchas veces son dignos de recordar los malos profesores, no solo porque no conocían la materia sino también por la forma de enseñarla. Recuerdo mucho durante la carrera de Derecho. Había muchos que ocupaban altos cargos en las magistraturas de los tribunales y de las Cortes, pero eran pésimos profesores. Parecía que su preocupación era la de "rajar" los estudiantes. Pensaban que mientras menos estudiantes pasaran la asignatura, eran mejores. El problema del Derecho consistía en que se nombraban profesores que podían conocer el Derecho, pero "no tenían vocación de docentes". La enseñanza del Derecho se convirtió para mí en una mera transmisión de conocimiento, en el sentido de recitar normas de memoria, pero con muy poca formación del jurista.

Según este relato, el buen maestro se caracteriza, entre otras cosas, por sus actitudes, por su dominio sobre el saber disciplinar y el conocimiento pedagógico. PM3 destaca de sus profesores memorables la singularidad en su labor docente; veamos a manera de ejemplo como:

Los profesores y el colegio INEM donde estudié, el cual tenía una modalidad de grado que se denominaba "Humanidades", nosotros vimos espacios académicos independientes tales como: Filosofía, Español, Taller Literario, Taller Artístico, Instituciones Sociales, Políticas y Económicas, Sociología, una materia dedicada al análisis de problemas desde la perspectiva jurídica, que no recuerdo su nombre, todas ellas materias con una importante dedicación semanal; por ejemplo, español podría tener cuatro horas a la semana, e independientemente taller literario, con un igual número, es decir, la "formación era excelente", si uno lo ve desde el punto de vista humanista y social. 


\section{¿De QUÉ MANERA EL}

CONOCIMIENTO SOBRE LA

DIDÁCTICA INCIDE EN LA

PRÁCTICA DE LOS PROFESORES

MEMORABLES?

Frente a esta pregunta, Porta (2010, p. 24) retoma a Litwin (2004), que afirma:

Hoy podemos reconocer tres orientaciones distintivas que fueron, en las últimas tres décadas inspiradoras de los estudios didácticos y desde las que los docentes pudimos construir perspectivas diferentes: pensar la clase anticipadamente reflexionar en torno a ella una vez concluida y actuar en ella con sabiduría (p.10).

Los docentes memorables narrarán sus experiencias para ir identificando el manejo de la didáctica.

PM1. Los aprendizajes los realicé con la práctica constante de mis "métodos pedagógicos", además con las orientaciones de personas idóneas, como los franciscanos con quienes me forme, además con dedicación y con mucha lectura tanto de libros de pedagogía como de didáctica.

PM2 destaca que dejó huella en él, la preocupación por la planificación de sus clases:

Recuerdo que había que llenar previamente lo que se conocía con el nombre de los "parceladores de clase" y terminada la clase había que llenar otro instrumento que correspondía a lo que se había dictado en la clase, llamado "El diario". Planificaba mis clases, para el semestre o lo del año de acuerdo con el número de horas para cada materia y para cada curso. Por lo general, había un texto y se seguía, casi al pie de la letra lo que decía el texto. El profesor prácticamente repetía el texto en clase y añadía algunos ejercicios que ayudaban al estudiante a grabar en su memoria lo aprendido, se hacían algunos ejercicios y cada tanto se hacía una "previa" o examen para constatar lo aprendido.

Por su parte, PM2 prefiere caracterizar ese desempeño en el escenario de la clase como su utilización de lo que llama "recursos didácticos", pero que evidentemente son parte de toda una producción docente.

Para PM3, algunas influencias para la vida profesional fueron:

En filosofía existe la práctica de escribir pequeños documentos que cumplen la función de "preparación de clase" a partir de los cuáles se desarrollan las sesiones. En un doble ejercicio: formación académica institucionalizada, pero también de una manera importante juega un papel la capacidad "autodidáctica".

Para Jackson (citado en Litwin, 2004, p. 13), la buena enseñanza no corresponde a una única manera de actuar, sino a muchas. Por qué como elegimos una estrategia, un modo de explicación, un tipo de respuesta, sigue siendo un interrogante a la hora de analizar las prácticas de los docentes.

En cuanto al conocimiento de la didáctica, PM4. dice que sus primeras clases las planificó con los conocimientos adquiridos, libros, parceladores, en el contexto. Lo cual nos hace ver que la creatividad juega un papel muy importante en el momento de enseñar, además de la influencia recibida por teóricos y algunos profesores mentores memorables propios de su época de formación. 


\section{¿QUÉ IMPACTO ACADÉMICO SE BUSCA EN LOS ESTUDIANTES POR PARTE DE UN PROFESOR MEMORABLE?}

Los docentes memorables buscan dejar huella en los estudiantes desde sus prácticas profesionales, tal como lo plantea PM4:

Creo, en esta etapa de mi vida, que la docencia es un saber, un arte un acto ético del saber y la persona que lo emprende. Creo que en las escenas educativas de enseñanza lo que hay es un diálogo permitido por la personalidad del maestro, sus discurso, su estilo y su conocimiento. Pero también hay un acto de validación social de ese diálogo que tiene que ver con la forma como el docente inicia la cátedra y como seduce a los estudiantes para que participen de ese discurso desde el punto de vista del interés y la conmoción. Enseñar es un ritual donde se coloca en contacto al estudiante con el saber tradicional y con sus preguntas y utilidades a la vida humana. Cuando enseño transmito saber, pasión, valores. El currículo y las didácticas son mediaciones que me ayudan pero mi interés no está solo ahí, está especialmente en ver cómo llego a las mentes de esos estudiantes y cómo posibilito que se expresen y crezcan humanamente y como pensadores. He tenido todo tipo de estudiantes y en general logro entrar en contacto con ellos porque no pienso en evaluarlos, ni en crecerme ante ellos, ni en que terminen sometidos, pienso en que compartan las dudas del pensamiento y se comprometan con comprender que uno se libera como ser humano cuando piensa. Nunca he necesitado herramientas de evaluación a modo instrumental en mi trabajo porque considero que todos los seres humanos pueden aprender si están de forma presente y consciente en estos procesos.
A su vez, PM3 dice que: "para mí la manera de impactar en mis estudiantes es dedicar más tiempo a la investigación y menos a la gestión administrativa".

De la misma manera, PM2 afirma que para lograr impacto en el rendimiento de los alumnos: "se deben mejorar las prácticas, fortaleciendo la investigación y profundizando más en las disciplinas".

En el caso de PM1, "el hacer intercambios académicos, participar en más seminarios y congresos nacionales e internacionales, es un aspecto fundamental".

Las diferentes experiencias resaltadas por cada uno de los profesores memorables objeto del estudio, apuntan a generar procesos de investigación, desde sus concepciones de formación permanente, de su dedicación a su quehacer pedagógico deslindándolo de lo que ellos consideran el quehacer administrativo. De la misma manera, todos fortalecen de muchas maneras, los procesos de producción científica, logrando el reconocimiento de su reflexión y de su actuar profesional.

\section{Discusión}

En esta investigación se resalta el valor de los relatos, expresados por los docentes memorables, para destacar la importancia de la didáctica en su actuar pedagógico como una de las características que hacen parte de la identidad profesional del profesor memorable con sus buenas prácticas de la enseñanza. Lo plantea Goodson, al referirse a las experiencias de vida, los docentes son: "ingredientes clave del individuo que somos o de lo pensamos acerca de nosotros mismos" (Goodson, 2003, p. 748) y esto tiene una fuerte marca en la forma en que 
transformamos nuestro quehacer en la enseñanza. Por eso se retoma el planteamiento hecho por Porta y Álvarez (2010), frente al pensamiento de Litwin (2008):

El relato de experiencias pasadas, su análisis y sus significados, las relaciones entre las experiencias pasadas y las presentes liberan a la imaginación y permiten una visión enriquecida del pasado. También los relatos heroicos de los próceres de nuestro pasado histórico, además de formar la ciudadanía, imperan modelos de vida y de conducta dotando de nuevos sentidos a los actos cotidianos (p. 19).

Volver sobre los sellos dejados por los profesores memorables, demostrarlos a la luz de las experiencias docentes, nos ayuda a reformular el estudio de la didáctica para luego influirlo en la práctica docente. Tenemos mucho que aprender de los profesores memorables en relación con la "buena práctica", la cual se articula con el concepto de "disposiciones didácticas". Desde la perspectiva de la reflexión y la comunicación, Litwin (1997) ha estudiado variaciones narrativas de las clases expositivas de docentes y construido con ellas una serie de ordenaciones didácticas. La idea de ordenación hace referencia a un conjunto inacabado, en construcción en el arte de narrar. Entendemos por ordenación didáctica: "la manera particular que despliega el docente para favorecer los procesos de construcción del conocimiento" (Litwin, 1998, p. 157). Esto implica la forma como el profesor memorable ejerce su actividad pedagógica, de su disciplina a través de los contenidos, para desarrollar sus procesos de enseñanza.

Los docentes memorables son fuente de inspiración. Intentamos tomar de ellos la sabiduría de sus explicaciones, el dominio de los contenidos, las estrategias apropiadas para cada situación. Como afirma Bain (2007) sobre los "mejores profesores universitarios", ellos suelen "desafiar intelectualmente a los estudiantes" (p. 39), enfrentando a los estudiantes a los problemas para poner a prueba sus problemas mentales, dándoles herramientas para que los reestructuren o los desarrollen. Podemos presentar algunas de las características que se definieron sobre los profesores memorables a partir de las entrevistas realizadas a los cuatro profesores memorables del Doctorado en Educación de la Universidad Santo Tomás.

"La importancia de los maestros en la primaria y en el bachillerato, al igual que la influencia de los padres de familia" (PM1, PM2, PM3, PM4), hizo que llegaran a elegir las carreras docentes que hoy ejercen con gran entusiasmo y responsabilidad.

"La profundización en la pedagogía" ha permitido que la enseñanza haya cambiado las técnicas, puesto que los medios y mediaciones son diferentes. La idiosincrasia del estudiante, así como sus intereses y necesidades han evolucionado. "Pero también personalmente han ocurrido cambios en tanto he adquirido mayor experiencia y muchos más conocimientos disciplinares y pedagógicos" (PM2).

"Uso de recursos en el ejercicio de su disciplina" (PM1, PM2, PM3), estos profesores demuestran que el conocimiento es construido, donde el interés es perentorio.

Los mejores profesores de universidad crean lo que podríamos llamar un entorno para el aprendizaje crítico natural, en el que incluyen las destrezas y la información que ellos quieren enseñar mediante trabajos que los estudiantes encontrarán fascinantes [...] Dejan de 
ser físicos aristotélicos y se convierten en newtonianos, porque han tenido el interés suficiente como cuestionarse a sí mismo (Bain, 2007, p. 59).

"La virtuosidad" es elemento clave para los docentes memorables (PM1, PM2, PM3, PM4) y hacen parte de las experiencias de vida y las referencias descritas incluso por Goodson (2003, pp. 744-748), de la misma manera, son aspectos claves de lo que piensan sobre sí mismos y sobre la forma como se enseña a partir de experiencias y antecedentes exitosos; esto es lo que se constituye en términos finales en las buenas prácticas de los docentes memorables. Otra característica importante de un profesor memorable universitario (PM1, PM2, PM3, PM4) es el "ejercicio del docente", donde el profesor se convierte en gestor de la reciprocidad educativa ya que no solo transmite conocimientos sin que juegue un papel importante en la manera de abordar este. Allí anida la importancia de la investigación unida a las "buenas prácticas": "hay que describir lo que caracteriza a las prácticas cuando las cosas funcionan bien, cuáles son las prácticas que son dignas de que se las tenga como modelo" (Schön, 1992, p. 11). Esta investigación continúa en la línea de organización y gestión escolar, intentando dar respuesta a temas de calidad docente a fin de conseguir la narración de experiencias de profesores memorables para ser reflexionadas y sometidas a juicio de expertos y para ser compartidas en un ambiente académico entre colegas.

\section{ReFERENCiAS}

Abdoulaye, A. (2003). Conceptualization Et Dissemination Des «Bonnes Pratiques» En Éducation: Essai D'une Approche Internationale À Partir
D'enseignements Tirés D'un Projet. Recuperado de http://portal.unesco. org/education/en/file_download.php/ f1685fde2633dd9b3b20fd828d6bfa92abdoulaye.pdf

Álvarez, I. (2009). Manual de narrativa televisiva. Córdoba, Argentina: Babel.

Álvarez, Z. y Porta, L. (2008). Biografías memorables: relatos sobre buenos profesores. En Porta, L. y Sarasa, M. C. Formación y desarrollo de la profesión docente en el profesorado: las buenas prácticas y sus narrativas. Mar del Plata: UNMDP Gráfica Tucumán.

Álvarez, Z. y Porta, L. (2010-2011). Una exploración del rol de los mentores en las trayectorias profesionales de los buenos docentes universitarios. Revista Praxis Educativa, XVI(14), 42-48.

Alves de Mattos, L. (1963). Compendio de la didáctica general. Buenos Aires: Kapeluz.

Arfuch, L. (2008). El espacio biográfico. Dilemas de la subjetividad contemporánea. Buenos Aires: Fondo de Cultura Económica.

Bain, K. (2007). Lo que hacen los mejores profesores universitarios. Valencia: Universidad de Valencia.

Benedito, V. (1987). Introducción a la didáctica, fundamentación didáctica y diseño curricular. Barcelona: Barcanova.

Bentolilla, S. (2002). Didáctica para los formadores de formadores. Un desafío en construcción. Revista Alternativas, Serie Espacios pedagógicos, 7(29), 159-174.

Bolívar, A. (2001). La investigación biográfica narrativa en educación. Enfoque y metodología. Madrid: La Muralla.

Bolívar, A. y Domínguez, J. (1998). Historia institucional de un centro escolar en 
organización y gestión de centros educativos. Barcelona: Praxis.

Bolívar, A. et ál. (1998). La investigación biográfico-narrativa en educación. Guía. Passim. Recuperado de http://www. google.com.co/url?sa=t\&rct=j\&q=\&esr $\mathrm{c}=\mathrm{s} \&$ source $=$ web\&cd $=2 \&$ ved $=0$ CFAQ FjAB\&url=http $\% 3 \mathrm{~A} \% 2 \mathrm{~F} \% 2 \mathrm{Fwww} . u g r$. es\%2F abolivar\%2FPostgrado1.doc\&e $\mathrm{i}=$ dgccULbRJsPJ6wGNkIHADw\&usg= AFQjCNHPLQY0pP8wD4qe9dvygMH on0FF2g

Bolívar, A.; Domínguez, J. y Fernández, M. (2001). La investigación bibliográfica narrativa en educación. Enfoque y metodología. Madrid: La Muralla.

Bolívar, A.; Domínguez, J. y Fernández, M. (2001). La investigación biográfica y narrativa en Iberoamérica. Campos de desarrollo y estado actual, 7(4), art. 12.

Booth, T. (1996). Sounds of still voices: issues im the use of narrative methods with people who have leaning difficulties. En Barto, L. (Ed.). Disability and Society: Emerging Issues and Insights. Nueva York: Logman Publishing.

Brannan, T.; Durose, C.; John, P., y Wolman, H. (2006). Assessing Best Practice as a Means of Innovation. Presented at the Annual Conference of the Urban Affairs Association, Montreal, Canada. Recuperado de http://www.ipeg.org.uk/ papers/UAA $\% 20$ paperfinal $\% 2017 \% 20$ April\%2006.pdf

Bruner, J. (2003). La fábrica de historias. Buenos Aires: Fondo de Cultura Económica.

Buendía, J. (1998). El romanticismo andaluz en su vertiente popular (vol. 3). Jaén, España: Universidad de Jaén.

Buendía, L.; Colas, P. y Hernández, F. (1998). Métodos de investigación en psicopedagogía. Madrid: McGraw-Hill.
Bunge, M. (1969). La investigación científica. Barcelona: Ariel.

Bunge, M. (1972). Teoría y realidad. Barcelona: Ariel.

Bunge, M. (1980). Epistemología. Barcelona: Ariel.

Bunge, M. (1985a). Seudociencia e ideología. Madrid: Alianza.

Bunge, M. (1985b). Racionalidad y realismo. Madrid: Alianza.

Chickering, A. y Gamson, F. (1987). Seven Principles for Good Practice in Undergraduate Education. Recuperado de http:// www.nnmc.edu/academics/assessment/ documents/sevenprinciples.pdf

Connelly, F. M. y Clandinin, D. J. (1994). The sage handbook of curriculum and instruction. California: Sage Publications.

Connelly, F. M. y Clandinin, D.J. (1994). Telling teaching histories. En Teacher Education Quarterlei, 2(1). San Francisco.

De Camilloni, A. R. W.; Cols, E.; Basabe, L. y Feeney, S. (2008). El saber didáctico. Buenos Aires: Paidós.

De Pablos, J. y González, T. (2007). Políticas educativas e innovación educativa apoyada en Tic: sus desarrollos en el ámbito autonómico. Presented at the II Jornadas Internacionales sobre Politicas Educativas para la Sociedad del Conocimiento, Granada, España. Recuperado de http://www.juntadeandalucia. es/averroes/jornadas_internacionales/ docs/upload/1101/1101C.pdf

Delacôte, G. (1997). Enseñar y aprender con nuevos métodos. La revolución cultural de la era electrónica. Madrid, España: Gedisa.

Díaz, Á. (2009). Pensar la didáctica. Buenos Aires - Madrid: Amorrortu. 
Dillard, A. (1982). Living by Fiction. Nueva York: Harper \& Row.

Donald, S. (1992). Una práctica profesional reflexiva (2aㅡ. ed.). Barcelona: Paidós.

Ettling, D. (1998). Levels of listening. En Braud, W. y Anderson, R.: transpersonal research methods for the social sciences. Londres: Sage publications.

Fenstermacher, G. D. (1989). Tres aspectos de la filosofía de la investigación sobre la enseñanza. En Wittrock, M. La investigación de la enseñanza. Barcelona: Paidós.

Fenstermacher, G. D. y Richardson, V. (1993). The Elicitation and Reconstruction of practical's arguments in teaching. Journal of curriculum studies, 25(2). Madrid: Morata.

Fenstermacher, G. D. y Richardson, V. (2005). On Makin. Determinations of Quality in Teaching. Journal Teacher's College Record: Columbia University: TC. Record núm. 1.

García, A. (2001). Didáctica universitaria. Madrid: La Muralla.

Goodson, I. (2003). Hacia un desarrollo de las historias personales y profesionales de los docentes. Revista Mexicana de Investigación Educativa, núm. 19. México: Consejo Mexicano de Investigación Educativa.

Guyot, V. (2007). Las prácticas del conocimiento. Un abordaje epistemológico: educación, investigación, subjetividad. San Luis, Argentina: Ediciones LAER, Universidad de San Luis.

Hernández, F. (1998). Historia oral y educación artística. En Hernández, F. y Ricart, M. (Eds.). III Jornadesd'Historia de l'Educación Artística. Barcelona: Facultat de Belles Arts.
Hernández, N. y Selva Chirico (2004). Ana Packer, construyendo saber y hacer enfermo. Montevideo: Trilce.

Imídeo, N. (1985). Metodología de la enseñan$z a$. Madrid: Kapeluz.

Klingberg, L. (1972). Didáctica general, contribuciones científicas de la pedagogía Alemana. Quito: ABYA-YALA.

Labarrere, R. G. y Valdivia, G. (1988). Pedagogía. La Habana: Editorial Pueblo y Educación.

Litwin, E. (2008). El oficio de enseñar. Condiciones y contextos. Buenos Aires: Paidós.

López, M. (2006). Una filosofía humanista de la educación. México: Trillas.

Malagón, J. L. (1999). Fundamentos del trabajo social comunitario. Bases teóricas y metodológicas para la intervención comunitaria. Sevilla: Aconcaya.

Pere, G. (2002). Las buenas prácticas. Barcelona: Universidad Autónoma de Barcelona - DIM.

Pérez, A. (1978). Las fronteras de la educación. Madrid: Zero.

Pérez, A. (1982). Memoria de oposiciones a cátedra de didáctica. Inédita.

Porta, L. (2010). Docencia universitaria. Propuesta para trabajar en el aula universitaria. Mar del Plata: Ediciones Suárez.

Porta, L. y Sarasa, M. C. (2009). Concepciones de la buena enseñanza: una aproximación narrativa. En XVI Encuentro estado de la investigación educativa: formación docente a debate, REDING, Universidad Católica de Córdoba, Facultad de Educación y CIFE/Reduc.

Porta, L. y Sarasa, M.C. (Comps.) (2008). Formación y desarrollo de la formación docente en el profesorado: las buenas prácticas y sus narrativas. Mar del Plata: Grupo de 
Investigaciones en Educación y Estudios culturales (G.I.EE.C)/ Facultad de Humanidades, Universidad de Mar del Plata. Argentina.

Ricoeur, P. (1995). Tiempo y narración: Vol. I; Configuración del tiempo; Vol. II, Configuración del tiempo en el relato de ficción; Vol. III, El tiempo narrado. México: Siglo XXI.

Sarasa, M. C. (2008). En torno a los modelos de buena enseñanza. En Formación $y$ desarrollo de la profesión docente en el profesorado: las buenas prácticas y sus narrativas. Mar del Plata: UNMDP Gráfica Tucumán.
Sarason, S. (2002). La enseñanza como arte de representación. Buenos Aires: Amorrortu.

Schulman, L. (1986). Those Who Understand: Knowledge Growth in Teaching. Journal Educational Researcher, núm. 2.

Steiner, G. (2004). Lecciones de los maestros. México: Siruela - Fondo de Cultura Económica.

Zabalza, M. (2004). A Didáctica univeresitaria. Un espazo disciplinar para o estudo e mellora da nosa docencia. Santiago de Compostela, España: Universidad de Santiago de Compostela. 